\title{
Laboreal
}

Volume $8 \mathrm{~N}^{\circ} 2$ | 2012

A antropotecnologia, ferramenta ou engodo?

\section{Inovação organizacional : sistemas de atividade, construção de redes e práticas associadas à adoção de tecnologias de gestão na administração pública chilena}

Innovación organizacional : sistemas de actividad, construcción de redes y prácticas asociadas a la adopción de tecnologías de gestión en la administración pública chilena Innovation organisationnelle: systèmes d'activité, construction de réseaux et pratiques associées à l'adoption de technologies de gestion dans l'administration publique au Chili Organizational innovation: activity systems, creating networks and practices associated with the adoption of management technologies in the Chilean public administration

\section{Gloria Zavala}

Tradutor. Ricardo Vasconcelos.

\section{OpenEdition}

Journals

\section{Edição electrónica}

URL: http://journals.openedition.org/laboreal/6818

DOI: 10.4000/laboreal.6818

ISSN: 1646-5237

\section{Editora}

Universidade do Porto

\section{Refêrencia eletrónica}

Gloria Zavala, «Inovação organizacional : sistemas de atividade, construção de redes e práticas associadas à adoção de tecnologias de gestão na administração pública chilena », Laboreal [Online], Volume 8 No2 | 2012, posto online no dia 01 dezembro 2012, consultado o 24 setembro 2020. URL: http://journals.openedition.org/laboreal/6818; DOI : https://doi.org/10.4000/laboreal.6818

Este documento foi criado de forma automática no dia 24 setembro 2020.

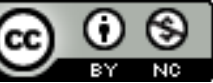

Laboreal está licenciado com uma Licença Creative Commons - Atribuição-NãoComercial 4.0 Internacional. 


\section{Inovação organizacional : sistemas de atividade, construção de redes e práticas associadas à adoção de tecnologias de gestão na administração pública chilena}

Innovación organizacional : sistemas de actividad, construcción de redes y

prácticas asociadas a la adopción de tecnologías de gestión en la administración pública chilena

Innovation organisationnelle: systèmes d'activité, construction de réseaux et pratiques associées à l'adoption de technologies de gestion dans l'administration publique au Chili

Organizational innovation: activity systems, creating networks and practices associated with the adoption of management technologies in the Chilean public administration

\section{Gloria Zavala}

Tradução : Ricardo Vasconcelos.

\section{REFERÊNCIA}

Zavala, G. (2012). Innovación organizacional : sistemas de actividad, construcción de redes y prácticas asociadas a la adopción de tecnologías de gestión en la administración pública chilena. Tesis de Doctorado, Facultad de Ciencias Sociales, Universidad de Chile, Santiago/Chile. 


\section{NOTA DO EDITOR}

Manuscrito recebido em : setembro/2012

Aceite após peritagem : novembro/2012

\section{Introdução : Mudanças na gestão pública}

1 Realizou-se um estudo de caso longitudinal no Fondo de Inversión Social (FOSIS), organismo dependente do Ministério de Planificação do Governo do Chile, com a finalidade de estudar processos de adoção de tecnologias de gestão. $O$ caso construiu-se considerando os anos de 2008 a 2010, em torno da implementação de um sistema informático de gestão de recursos humanos (SIGestiona) e do leventamento de competências e construção de novos perfis de cargo.

2 Nos últimos anos as organizações públicas chilenas iniciaram um processo de modernização dos seus sistemas de gestão (Ramírez, 2002 ; Waissbluth \& Inostroza, 2007 ; Waissbluth, 2008). Este olhar vem de mãos dadas com as abordagens de um grupo não sistemático de soluções à burocracia tradicional, chamado Nova Gestão Pública (NGP). E que, apesar das críticas, continua a manter-se como um pilar na orientação no sentido de uma maior eficiência das organizações no âmbito público (Waissbluth \& Inostroza, 2007).

\section{Problema}

3 É dentro deste olhar que a adoção de inovações ao nível da gestão alcançou grande relevância (Damanpour, 1991; 1996; Goya, 2002 ; Ramírez, 2002 ; Falivene \& Silva, 2005 ; Bastidas \& Moreno, 2007; Damanpour \& Wichnevsky, 2006; Damanpour \& Schneider, 2008).

Diaz (2010) sugere que a inclusão de diversos paradigmas de gestão incentivou formas de construção organizacional com as seguintes características (Díaz, 2010, pp. 21-22) : Incorporação de "sistemas tecnológicos e organizacionais (...) com fortes descontinuidades quanto à sua filiação histórica (...) ; fragmentação funcional, social e cultural (...) ; dissociação do nexo no interior da cadeia missão, visão, estratégia, processos, estrutura (...)".

5 Isso dificultaria o surgimento de representações baseadas em atividade funcional compartilhada, requisito para a flexibilidade orgânica que supõe a NGP.

6 Considerando esta problemática, uma análise da literatura a nível latino-americano e nacional, não dá conta de maiores desenvolvimentos em torno das dinâmicas internas que acompanham os processos de adoção de inovações de gestão, tendo em conta a sua sequência temporal (Rainieri, 1998; Goya, 2002 ; Waissbluth \& Inostroza, 2006, 2007 ; Waissbluth, 2008). 


\section{Objectivo e quadro de análise conceptual}

7 O estudo visou analisar, com um enfoque em sistemas de atividade de redes, os processos de adoção de novas tecnologias de gestão na Subdireção de Pessoas (SDP) do Fondo de Inversión Social (FOSIS), uma organização da administração pública chilena.

Nesta base, para a realização da análise diferenciaram-se os seguintes pontos :

1. Propósitos : construção de propósitos a partir dos discurss dos membros da SDP.

2. Sistemas de atitivade (Cole \& Engeström, 2001; Engeström, 1987; Engeström, 2003; Engeström \& Escalante, 2001). Implicando pontos de conflito e solução dentro e entre os sistemas de atividade, cilcos de aprendizagem e desenvolvimento de competências, integrando aspetos das perspetivas de Cognição Distribuída (Hutchins, 1995 ; Salomon, 1993) e Ação Situada (Suchman, 1987, 2007).

3. Competências, aprendizagem (Diaz, 2000, 2008), quadro tecnológico e mecanismos de fecho dos grupos sociais relevantes (Pinch \& Bijker, 1984).

4. Práticas de Construção de redes : entre sistemas de atividade e a partir da Teoria do AtorRede (Calon, 1986).

\section{Quadro metodológico}

9 Para alcançar estes objetivos, levou-se a cabo um estudo de caso qualitativo, descritivo e longitudinal.

Os dados para a construção do estudo foram produzidos através de observação, análise documental e entrevistas abertas semi-diretivas (Ortí, 1992) aos membros da SDP e cobrem o período de 2008 a 2010.

11 Utilizou-se análise de discurso e análise de conteúdo qualitativo.

\section{Resultados}

12 Ao nível do contexto institucional, quadro tecnológico e grupos sociais relevantes em torno da adoção tecnológica, identificaram-se três tipos de propósitos :

1. Propósito tradicional-normativo : cumprimento de normas da Administração Pública.

2. Propósito técnico-instrumental : busca da eficiência e da eficácia na gestão.

3. Propósito administrativo-político : melhora a imagem interna da SDP.

13 A organização pública estrutura-se principalmente a partir de um propósito tradicional-normativo, em todas as suas unidades, ainda que com graus de prioridade distintos, já que só se vê resguardada a partir deste propósito quanto à sua existência e permanência no tempo. Neste sentido, por exemplo, a informatização relaciona-se com o desencadear de fortes investimentos associados a convénios de desempenho.

14 A construção de redes dá-se a partir de processos políticos orientados por diversos propósitos, observando-se uma ampla motivação por grupos principalmente profissionais (em contraste com técnicos e administrativos) pelo cumprimento de propósitos instrumentais. No entanto, é a lógica burocrática, tradicional-normativa, a única que acaba por manter o imperativo de continuar com o uso de novas tecnologias, principalmente quando estas ficam sem porta-voz. 
Neste sentido, as expectativas de melhoria varia amplamente quando se estabelece um contraste com o que realmente os artefactos podem aportar ao trabalho quotidiano, gerando-se ajustes ad-hoc, com a intenção de integrar de alguma forma as novas tecnologias, sem se sair de um quadro de representações comuns da atividade, implicando um esforço de constante negociação sociotécnica por parte dos membros da organização.

16 As possibilidades de aprendizagem neste contexto parecem ser limitadas devido à falta de adequação dos artefactos às formas de fazer o trabalho, gerando-se ciclos de solução de problemas contingentes, orientados para o cumprimento das tarefas e para a sua consequente permanência dentro da lógica organizacional (considerando principalmente que a coordenação de base é burocrática), sem deixar espaço à inovação.

\section{Conclusões}

17 A utilidade dos instrumentos de gestão, tanto informáticos como não informáticos, como no caso das competências, está em conseguirem constituir-se objetos limite, em vez de pontos de passagem obrigatória, artefactos estabilizados. Isto faz referência à construção de uma intersubjetividade, implicando a consideração da ação como situada, num cenário que dá sentido às variações do contexto no qual se articula a atividade.

Consequentemente, considera-se que os limites de um artefacto tecnológico nunca são apenas físicos, são conceptuais, práticos e políticos. Dentro de uma negociação constante de significados nunca estáveis, nunca univalentes, nunca claros, tanto em termos das interpretações associadas ao artefacto, ao processo ou aos grupos participantes.

\section{BIBLIOGRAFIA}

Bastidas, E., \& Moreno, Z. (2007). El cuadro de mando integral en la gestión de las organizaciones del sector público : Universiad Centroccidental Lisandro Alvarado. Compendium, 10 (018), 5-20. Consultado en octubre, 07, 2010 de http://redalyc.uaemex.mx/pdf/880/ 88001802.pdf

Callon, M. (1986). Some elements of a sociology of translation : domestication of the scallops and the fishermen at St Brieuc Bay. In John. Law (Ed.), Power, action and belief: a new sociology of knowledge? (pp. 196-223). London : Routledge.

Cole, M., \& Engeström, Y. (2001). A cultural-historical approach to distributed cognition. In Gavriel Salomon (Ed.), Distributed Cognitions : Psychological and Educational Considerations (pp. 1-46). New York : Cambridge University Press.

Damanpour, F. (1991). Organizational innovation : a meta-analysis of effects of determinants and moderators. Academy of Management Journal, 34 (3), 555-590. 
Damanpour, F. (1996). Organizational complexity and innovation : developing and testing multiple contingency models. Management Science, 42 (5), 693-716.

Damanpour, F., \& Schneider, M. (2008). Characteristics of innovation and innovation adoption in public organizations : assessing the role of managers. Journal of Public Administration Research and Theory, 19 (3), 495-522.

Damanpour, F., \& Wischnevsky, J. D. (2006). Research on innovation in organizations : Distinguishing innovation-generating from innovation-adopting organizations. Journal of Engineering and Technology Management, 23 (4), 269-291.

Díaz, C. (2000). Impacto Organizacional y en las Personas de las Implantaciones Tecnológicas : dos casos de referencia en Chile. In Actas ESOCITE, s/p, Sao Paulo.

Díaz, C. (2008). Actividad, contexto organizacional y competencias. Revista Psicología Organizacional Humana (UAI), 2, 53-68.

Díaz, C. (2010). Tensiones y proyecciones en la modernización de la gestión pública : discusión desde una perspectiva histórico-cultural. Psicoperspectivas, 9 (1), 7-28.

Engeström, Y. (1987). Learning by expanding : an activity theoretical approach to developmental research. Helsinki : Orienta-Konsultit Oy.

Engeström, Y. (2003). Activity theory and individual and social transformation. In Yrjö Engeström, Reijo Miettinen \& Raija-Leena Punamäki (Eds.), Perspectives on activity theory (pp. 19-38). New York : Cambridge University Press.

Engeström, Y., \& Escalante, V. (2001). Mundane tool or object of affection? The rise and fall of the Postal Buddy. In B. A. Nardi (Ed.), Context and Consciousness. Activity theory and human-computer interaction (pp. 325-373). Cambridge : The MIT Press.

Falivene, G., \& Silva, G. (2005). Formación, cambio tecnológico e innovación en organizaciones públicas. X Congreso Internacional del CLAD sobre la Reforma del Estado y de la Administración Pública, Santiago, Chile. Consultado en julio, 03, 2010 de http://www.sgp.gov.ar/contenidos/ag/paginas/ ponencias/docs/clad_x/

Formacion_cambio_tecnologico_innovacion_organizaciones_publicas.pdf

Goya, F. (2002). Innovación en la gestión pública chilena : dos enfoques alternativos. VII Congreso Internacional del CLAD sobre la Reforma del Estado y de la Administración Pública, Lisboa, Portugal. Consultado en julio, 03, $2008 \mathrm{de} \mathrm{http://unpan1.un.org/intradoc/groups/} \mathrm{public/documents/}$ CLAD/clad0043606.pdf

Hutchins, E. (1995). Cognition in the wild. Cambridge : The MIT Press.

Ortí, A. (1992). La apertura y el enfoque cualitativo o estructural : la entrevista abierta y la discusión de grupo. In Manuel García Ferrando, Jesús Ibañez \& Francisco Alvira (Comps.), El análisis de la realidad social : métodos y técnicas de investigación (pp. 189-221). Madrid : Alianza Universidad Textos.

Pinch, T., \& Bijker, W. (1984). The social construction of facts and artefacts : or how the sociology of science and the sociology of technology might benefit each other. Social Studies of Science, 14, 399-441.

Rainirei, A. (1998). Creencias y cambio organizacional en los sectores público y privado. Estudios Públicos, 70 (otoño), 201-229.

Ramírez, Á. (2002). Innovación en la gestión pública : lecciones, aprendizajes y reflexiones a partir de la experiencia chilena. VII Congreso Internacional del CLAD sobre la Reforma del Estado y de la 
Administración Pública, Lisboa, Portugal. Consultado en septiembre, 01, 2008 de http:// unpan1.un.org/intradoc/groups/public/documents/CLAD/clad0043607.pdf

Salomon, G. (1993). No distribution without individuals' cognition : a dynamic interactional view. In Gavriel Salomon (Ed.), Distributed Cognitions : Psychological and Educational Considerations (111-138). Ney York : Cambridge University Press.

Suchman, L. (1987). Plans and situated actions : the problem of human-machine communication. New York : Cambridge University Press.

Suchman, L. (2007). Human-machine reconfigurations. Plans and situated actions ( $2^{\circ} \mathrm{Ed}$.). New York : Cambridge University Press.

Waissbluth, M. (2008). Gestión del cambio en el sector público. Comparative and General Pharmacology, Santiago. Consultado en junio, 14, 2010 de http://old.dii.uchile.cl/boletin/ 20090331/_contenido/publicaciones/1/

Gestiooon_del_Cambio_en_el_Sector_Puuublico,_Mario_Waissbluth.pdf

Waissbluth, M., \& Inostroza, J. (2006). La reforma del Estado en Chile 1990-2005. De la confrontación al consenso. Barcelona : PUBLIC, Boletín del Instituto de Gobernanza y Dirección Pública de ESADE. Consultado en junio, 14, 2010 de http://www.esade.edu/public

Waissbluth, M., \& Inostroza, J. (2007). Globalización y reforma del Estado en Chile. Iberoamericana. Nordic Journal of Latinoamerican and Caribbean Studies, XXXVII (1), 285-310.

\section{AUTORES}

\section{GLORIA ZAVALA}

Universidad de Chile - Facultad de Ciencias Sociales, Departamento de Psicología, Av. Capitán Ignacio Carrera Pinto, no 1045 , Santiago de Chile gzavala@u.uchile.cl 